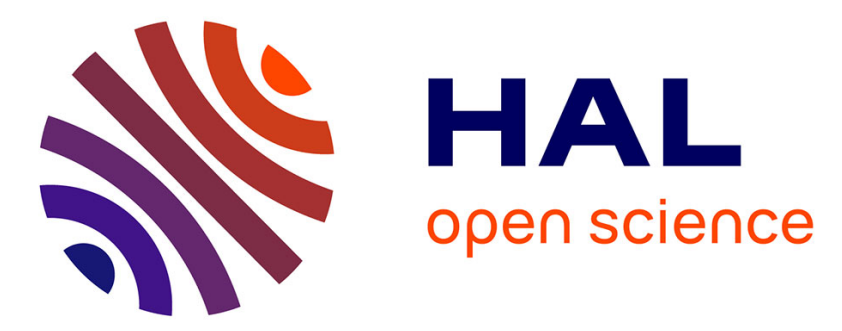

\title{
Coherent Wake Emission of High-Order Harmonics from Overdense Plasmas
}

\author{
F. Quéré, Cédric Thaury, P. Monot, S. Dobosz, Ph. Martin, J.-P. Geindre, P.
} Audebert

\section{> To cite this version:}

F. Quéré, Cédric Thaury, P. Monot, S. Dobosz, Ph. Martin, et al.. Coherent Wake Emission of High-Order Harmonics from Overdense Plasmas. Physical Review Letters, 2006, 96, pp.125004. 10.1103/PhysRevLett.96.125004 . hal-01166798

\section{HAL Id: hal-01166798 \\ https://hal.science/hal-01166798}

Submitted on 23 Jun 2015

HAL is a multi-disciplinary open access archive for the deposit and dissemination of scientific research documents, whether they are published or not. The documents may come from teaching and research institutions in France or abroad, or from public or private research centers.
L'archive ouverte pluridisciplinaire HAL, est destinée au dépôt et à la diffusion de documents scientifiques de niveau recherche, publiés ou non, émanant des établissements d'enseignement et de recherche français ou étrangers, des laboratoires publics ou privés. 


\title{
Coherent Wake Emission of High-Order Harmonics from Overdense Plasmas
}

\author{
F. Quéré, C. Thaury, P. Monot, S. Dobosz, and Ph. Martin \\ Service des Photons, Atomes et Molécules, Commissariat à l'Energie Atomique, DSM/DRECAM, CEN Saclay, \\ 91191 Gif sur Yvette, France \\ J.-P. Geindre and P. Audebert \\ Laboratoire pour l'Utilisation des Lasers Intenses, UMR 7605-CNRS-CEA-Ecole Polytechnique-Université Paris VI, \\ F91128 Palaiseau, France
}

(Received 9 January 2006; published 30 March 2006)

\begin{abstract}
We present a new mechanism for high-order harmonic generation by reflection of a laser beam from an overdense plasma, efficient even at moderate laser intensities (down to $I \lambda^{2} \approx 4 \times 10^{15} \mathrm{~W} \mathrm{~cm}^{-2} \mu \mathrm{m}^{2}$ ). In this mechanism, a transient phase matching between the electromagnetic field and plasma oscillations within a density gradient leads to the emission of harmonics up to the plasma frequency. These plasma oscillations are periodically excited in the wake of attosecond electron bunches which sweep across the density gradient. This process leads to a train of unevenly spaced chirped attosecond pulses and, hence, to broadened and chirped harmonics. This last effect is confirmed experimentally.
\end{abstract}

PACS numbers: 52.38.Kd, 42.65.Ky

High-order harmonic generation (HHG) by reflection of an intense laser beam from an overdense plasma has been observed with very different types of lasers, from nanosecond $\mathrm{CO}_{2}$ lasers to picosecond (ps) Nd-glass and femtosecond (fs) Ti-Sa lasers ([1-6], and references therein). Two strong motivations drive these studies. First, this is a possible path to generate energetic subpicosecond shortwavelength light pulses, which are of high interest for many research fields. Second, studying the properties of these harmonics should provide new insight into the dynamics of high-intensity laser-plasma interaction and, in particular, into the collective-absorption mechanisms leading to energetic electrons, which play a crucial role in effects such as incoherent x-ray emission, or ion acceleration from thin foils.

Most experiments on HHG from plasmas have so far been performed in, or very close to, the so-called relativistic intensity regime $I \lambda^{2}>1.37 \times 10^{18} \mathrm{~W} \mathrm{~cm}^{-2} \mu \mathrm{m}^{2}$, where the quiver motion of electrons in the laser field is relativistic. In this regime, the most common interpretation for the short-pulse experiments is provided by the oscillating mirror $(\mathrm{OM})$ model $[7,8]$ : The intense laser field drives a relativistic oscillation of the plasma surface, which in turn gives rise to a periodic phase modulation of the reflected beam and, hence, to the generation of harmonics of the incident frequency.

In this Letter, we demonstrate both theoretically and experimentally that harmonics up to the plasma frequency of the target can also be generated through a totally different mechanism, which we call coherent wake emission (CWE), efficient well below the relativistic regime. This mechanism is associated with plasma-wake oscillations in a density gradient and turns out to be a remarkably universal source of radiation in plasmas; the same process has recently been identified as a way to generate intense terahertz $(\mathrm{THz})$ pulses in inhomogeneous underdense plasmas excited by a laser wake [9]. In the present case, CWE is driven by energetic electron bunches generated around the critical density and which sweep across the overdense plasma. The periodic repetition of this mechanism, every optical cycle, leads to a train of attosecond (asec) pulses and, thus, to a spectrum consisting of harmonics of the incident laser frequency and extending up to the extreme ultraviolet. The importance of plasma oscillations excited by fast electrons has been pointed out recently in the context of HHG from the rear side of thin foils [10].

Our study was motivated by a striking experimental fact. Focusing a few millijoules from a $50 \mathrm{fs} 800 \mathrm{~nm} 1 \mathrm{TW}$ Ti-Sa laser (LUCA laser, Saclay Laser Interaction Center) on a fused silica target, at $45^{\circ}$ incidence and in $p$ polarization, we observed harmonics in the specularly reflected beam up to orders 16-18 [Fig. 1(a)], even for intensities down to $I \lambda^{2}=4 \times 10^{15} \mathrm{~W} \mathrm{~cm}^{-2} \mu \mathrm{m}^{2}$. In this intensity range, more than 2 orders of magnitude below the relativistic interaction regime, the $\mathrm{OM}$ mechanism has an extremely low efficiency [8] and is, thus, very unlikely to be responsible for such high-order harmonics.

We have measured the intensities of harmonics $12-14$ as a function of the laser intensity between $I \lambda^{2}=4 \times 10^{15}$ and $2 \times 10^{16} \mathrm{~W} \mathrm{~cm}^{-2} \mu \mathrm{m}^{2}$. The evolution of their efficiencies is presented in Fig. 1(b), which shows two essential features. First, these efficiencies vary very weakly with laser intensity $\left(\approx I^{0.4}\right)$. Second, this intensity dependence is almost the same for all harmonics, which means that the harmonic spectrum hardly changes with intensity. Both observations are in contradiction with the $\mathrm{OM}$ mechanism $[2,3,5]$, which predicts a highly nonlinear behavior in this intensity range. This definitely excludes this process in interpreting our observations.

To identify the generation mechanism, we have used the $1 \frac{1}{2}$-D relativistic particle-in-cell (PIC) code EUTERPE [11]. When simulating the interaction of a laser pulse at 

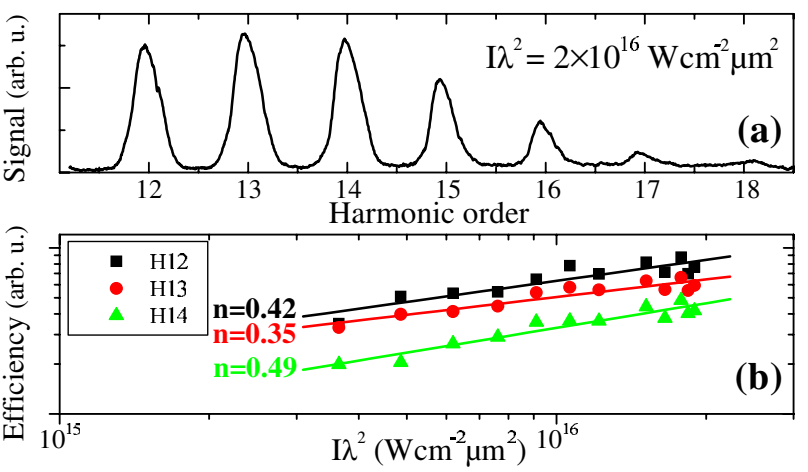

FIG. 1 (color online). (a) Typical raw harmonic spectrum; (b) conversion efficiency $I_{N} / I_{0}$ for harmonics $12-14$ as a function of laser intensity. The solid lines are power-law fits, which exponents are given on the graph.

$10^{16}-10^{17} \mathrm{~W} \mathrm{~cm}^{-2} \mu \mathrm{m}^{2}$ with a plasma of maximum density $n_{s}$, all harmonics up to the plasma frequency $\omega_{p}^{s}=$ $\left(n_{s} e^{2} / m \epsilon_{0}\right)^{0.5}$ are observed in the spectrum of the reflected beam, leading to a maximum harmonic order $N_{\max }=$ $\left(n_{s} / n_{c}\right)^{0.5}$, where $n_{c}$ is the critical density for the laser wavelength $\lambda$. At these intensities, $n_{s}$ is typically a few hundreds of $n_{c}$, and harmonic orders above 10 are therefore obtained, as in our experiment. Importantly, this HHG signal is obtained in the simulations only for oblique incidence and $p$ polarization, as observed experimentally. Besides, it occurs only when a short density gradient is present at the surface of the plasma, with a typical scale length $L$ between $\lambda / 50$ and $\lambda / 15$ at the intensities considered here. In the experiment, such a gradient most likely develops during the few ps preceding the main pulse, due to the rather high pedestal of the LUCA laser on the ps time scale ( $\approx 10^{-4}$ at 5 ps and $\approx 10^{-3}$ at $1 \mathrm{ps}$ ).

We now analyze in detail the results of the PIC simulations to get a physical understanding of the generation mechanism. We first specifically choose the parameters of the simulation (e.g., the laser intensity) to show that, even close to the relativistic regime, the OM mechanism is clearly not the only process leading to harmonic emission. Figure 2 shows a plot of the electronic density along the target normal $x$, as a function of time. Also shown on this graph is the envelope of the magnetic field $B_{z}$ corresponding to the superposition of harmonics 5-9, obtained by spectrally filtering $B_{z}(t)$ at each point of the spatial grid.

The emission of two separated asec pulses every optical cycle is observed on this graph. One of these pulses clearly originates from the laser-driven oscillations of the critical density surface; this corresponds to the OM generation process. The other asec pulse is generated in a much denser part of the plasma, where the laser pulse does not penetrate, and has to be attributed to a different mechanism. We note that the time spacing between these two pulses is not fixed and depends on the physical parameters. At lower intensities, such as those used in our experiment, the OM contribution becomes absolutely negligible, and the HHG signal is largely dominated by this second class of asec pulses. In this regime, a detailed analysis shows that the $N$ th harmonic is generated in the density gradient, around the point $x$ where the local plasma frequency $\omega_{p}(x)$ is of the order of the harmonic frequency. This suggests that plasma oscillations play a crucial role in the generation of these asec pulses and raises two questions. (i) How are these high-frequency plasma oscillations excited? (ii) How do they emit a single asec pulse every optical cycle?

Our simulations reveal that plasma oscillations are induced in the wake of energetic bunches of electrons, consistent with Ref. [10]. The formation of these bunches is observed to occur around $n(x)=4 n_{c}$ and around a given phase within each optical cycle. In Fig. 2, we intentionally used a thin plasma foil, so that these bunches can be observed when they exit the plasma at the rear side of the foil (white arrows). This reveals that their duration is very small, typically on the hundred asec time scale. These short bunches impulsively excite plasma oscillations all along the gradient. Their density and the amplitude of the induced plasma-wake oscillations are typically of the order of $n_{c}$.

The mechanism for the formation of these electron bunches can also be inferred from the PIC simulations. Since the laser is $p$ polarized, and we are in a regime where the quiver velocity in the laser field $v_{\text {osc }}$ is such that $v_{\text {osc }} / c \geqslant 2 \pi L / \lambda$, some electrons are accelerated in the underdense part of the plasma by vacuum heating [12]. As can be seen in Figs. 1(c)-1(d) in Ref. [12], a portion of these energetic electrons naturally bunches temporally as it penetrates the overdense part of the plasma. In our simulations, this bunching is observed to occur in the density gradient, leading to the efficient excitation of plasma oscillations therein.

We now use a simple analytical model, analogous to the one used in Ref. [9] for THz pulses, to show how these plasma-wake oscillations in a density gradient can emit bursts of light. We focus on what occurs during a single

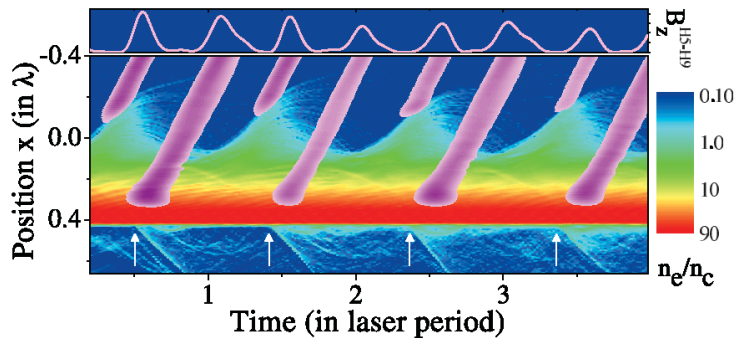

FIG. 2 (color). Electronic density in log scale and intensity of the generated asec pulses (purple color scale). The calculation was performed with an angle of incidence $\theta=45^{\circ}, n_{s}=80 n_{c}$, $L=\lambda / 15$, a foil thickness $d=\lambda / 15$, and a constant intensity of $4 \times 10^{17} \mathrm{~W} \mathrm{~cm}^{-2} \mu \mathrm{m}^{2}$. The incident laser field propagates from negative to positive $x$. The upper panel shows the temporal profile of the asec pulses at $x=-0.4 \lambda$. 
optical cycle of the laser and assume for simplicity that the energetic electrons propagate along the target normal, with a constant velocity $v<c$. An electron bunch, originating from $(0, y)$, then reaches the point $(x, y)$ in the plasma at time $t_{0}=y \sin \theta / c+x / v$. This equation defines a line (or a plane in 3D), corresponding to a density wave constituted of the energetic electrons, that sweeps along the target surface with the same phase velocity $c / \sin \theta$ as the laser field and along the target normal with the velocity $v$ of the individual electrons. This density wave, sketched in Fig. 3(a), triggers plasma oscillations in its wake, starting at $t=t_{0}(x, y)$ for any point $(x, y)$. In an exponential density gradient, where $n(x) \propto \exp (x / L)$ for $n \leq n_{s}$, the phase $\phi(x, y, t)$ of the resulting plasma-wake oscillations is thus given by

$$
\phi(x, y, t)=\omega_{p}(x)\left[t-t_{0}\right] \propto e^{x / 2 L}\left[t-t_{0}(x, y)\right] .
$$

Figure 3(a) shows the wave fronts of the resulting wakefield, at a given $t$. The wave vector of these plasma oscillations $\mathbf{k}=-\nabla \phi$ is a function of time and space, which is easily calculated using Eq. (1). This calculation shows that $k_{x}$ cancels along a line given at time $t$ by $y \sin \theta / c+x / v+$ $2 L / v=t$. This line, also shown in Fig. 3(a) (dashed line), is parallel to the density wave but shifted in time by $2 L / v$.

Along this line, the wave vector of the plasma-wake oscillations is $\mathbf{k}=\left(0, \omega_{p}(x) \sin \theta / c\right)$ : This is precisely the local wave vector, around its turning point, of a light wave with frequency $\omega_{p}(x)$, emerging from the density gradient with an angle $\theta$ [13]. This local phase matching between the plasma oscillations and the electromagnetic waves leads to a light emission localized along this line, due to the associated stationary-phase point in the spatial integral giving the electromagnetic field emitted by the plasma. This is the CWE process. The emitted wave is then refracted as it propagates down the density gradient

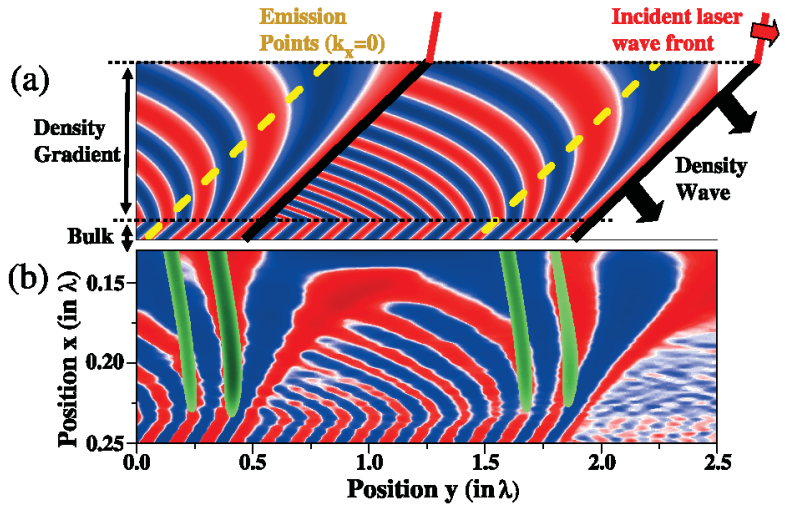

FIG. 3 (color). (a) Density wave sweeping across the density gradient and wave fronts of the resulting plasma-wake oscillations [i.e., contour lines of $\cos \phi(x, y)$ ]. (b) Wave fronts of $E_{x}$ (blue and red) and of the frequency-filtered $B_{z}$ (green), obtained from the PIC code for $I \lambda^{2}=10^{17} \mathrm{~W} \mathrm{~cm}^{-2} \mu \mathrm{m}^{2}, L=\lambda / 40$, $\theta=45^{\circ}, n_{s}=180 n_{c}$. and eventually emerges at an angle $\theta$ from the target normal, i.e., in the specular reflection direction.

More qualitatively, Fig. 3(a) shows that this emission line corresponds to the points where the wave fronts of the plasma oscillations bend and change direction. This bending occurs because plasma oscillations with increasing frequencies are excited at later times $t_{0}$ [i.e., in Eq. (1), $\omega_{p}$ increases with $x$, while $\left(t-t_{0}\right)$ decreases]. Physically, this is simply due to the electron bunch coming from the low density part of the gradient: The wave front bending, thus, directly derives from the geometry of the interaction and is bound to occur whatever the exact shape of the density gradient.

At the intensities considered in this Letter, $v$ is of the order of a few $10^{-1} c$. It therefore takes the electron bunch a fraction of the laser optical cycle to cross the density gradient. CWE then occurs at a given delay $2 L / v$ after the electron bunch has passed and lasts only for about one plasma oscillation period at each $x$; light is, hence, emitted in the form of asec pulses. Because the different frequencies making up this pulse are generated at different points along the gradient, this asec pulse is chirped, just like the THz pulses emitted by CWE in underdense plasmas [9].

Figure 3(b) now shows the results of a PIC simulation over a little more than one wavelength of the laser field along the plasma surface. Instead of plotting the density as in Fig. 2, we plot the electric field $E_{x}$, which enables us to visualize the plasma oscillations with much less noise. The wave fronts of the magnetic field $B_{z}$ associated to harmonics 7-13 are also shown: They clearly originate from the points where the wave fronts of the plasma oscillations bend. This fact, as well as the absolutely striking analogy between Figs. 3(a) and 3(b), unambiguously demonstrates that the asec pulses seen in the PIC code results are indeed generated by CWE.

CWE is a linear conversion process, which can to a certain extent be considered as the temporal reversal of resonance absorption [9]. Besides, the amplitude of the plasma-wake oscillations leading to CWE scales linearly with the charge of the driving electron bunches. In vacuum heating, this charge also scales linearly with the amplitude of the laser electric field. Within its range of validity, this generation mechanism thus has an essentially constant efficiency with laser intensity. In the PIC code results, it is typically of the order of $10^{-3}-10^{-4}$ for harmonic 10 . The weak variations observed experimentally [Fig. 1(b)] are most likely due to variations in the gradient scale length or ionization state of the plasma.

We now consider the temporal structure of the fs train of asec pulses generated during the whole laser pulse. According to the previous model, the time delay between the formation of the electron bunch at $n \approx 4 n_{c}$ and the subsequent generation of an asec pulse of central frequency $\omega_{N}$, occurring around $x_{N}=L \ln \left(\omega_{N}^{2} / 4 \omega_{L}^{2}\right)$, is given by $\beta L / v$, with $\beta=2+x_{N} / L$ and $\omega_{L}$ the laser angular fre- 


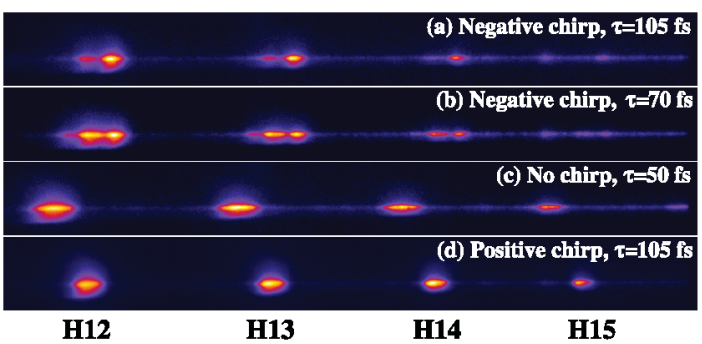

FIG. 4 (color). Normalized experimental spectral profiles of harmonics $12-15$ for different chirps of the incident laser pulse. The laser energy was kept constant and corresponds to $I \lambda^{2}=$ $3 \times 10^{16} \mathrm{~W} \mathrm{~cm}^{-2} \mu \mathrm{m}^{2}$ for a $50 \mathrm{fs}$ pulse.

quency. Three factors can, thus, affect the emission time of the asec pulse: The phase of the laser field at the time of creation of the electron bunch, the gradient scale length $L$, and the electron velocity $v$. PIC simulations suggest that the first two factors change very little during the laser pulse. Neglecting their variations, and assuming that $v$ varies as $v(t) \approx v_{0}\left(1-t^{2} / \tau^{2}\right)$ around the pulse maximum, the time spacing between successive asec pulses is then given within the analytical model by $\Delta t=T_{L}[1+$ $\left.2 \beta L t / v_{0} \tau^{2}\right]$. Hence, due to the changes in intensity during the fs laser pulse, this time spacing linearly increases during the laser pulse, in remarkable analogy with what occurs for HHG in gases [14].

Such an increasing time spacing results in a negative contribution to the chirp (i.e., blue precedes red) of the individual harmonics in the train spectrum [14], given for harmonic $N$ by $d \omega_{N} / d t=-2 \omega_{N} \beta L / v_{0} \tau^{2}$. We have, therefore, used the PIC code to calculate the chirp of the 7 th harmonic generated by a $40 \mathrm{fs} 800 \mathrm{~nm}$ laser pulse with $L=\lambda / 30, n_{s}=180 n_{c}$, and $I \lambda^{2}=10^{17} \mathrm{~W} \mathrm{~cm}^{-2} \mu \mathrm{m}^{2}$, and indeed obtained a negative chirp $d \omega_{N} / d t=$ $-0.02 \mathrm{PHz} / \mathrm{fs}$. This is in excellent agreement with the value obtained from the previous formula, using the velocity $v_{0}=c / 3$ provided by the PIC code results.

As already demonstrated for HHG in gases [15], such a negative chirp of the individual harmonics can be compensated by using a laser pulse with a positive chirp $d \omega_{L} / d t$ for the generation, such that $N d \omega_{L} / d t \approx-d \omega_{N} / d t$. Spectrally, this compensation, either complete or partial, leads to a narrowing of the harmonics. To test the above predictions experimentally, we have therefore measured HHG spectra for different chirps of the incident pulse (Fig. 4): Compared to the Fourier-transform limited case [Fig. 4(c)], a clear narrowing of the harmonic peaks occurs for the positive chirp [Fig. 4(d)], while the harmonics are even broader for a negative chirp of the same magnitude [Fig. 4(a)]. Spectral shifts are also observed: Although this can be expected, they are difficult to predict quantitatively, since they depend on how the density gradient and its temporal dynamics evolve with the pulse duration and intensity. From these results, the chirp of these harmonics can be estimated to be of the order of $10^{-2} \mathrm{PHz} / \mathrm{fs}$ for a 50 fs laser pulse at $3 \times 10^{16} \mathrm{~W} \mathrm{~cm}^{-2} \mu \mathrm{m}^{2}$. Both the sign and the order of magnitude of this chirp are consistent with the results of the PIC simulations. This is strong evidence that the harmonics observed in our experiment are indeed produced by the CWE mechanism, triggered by lasergenerated asec electron bunches.

In conclusion, we have demonstrated theoretically and experimentally that high-order harmonics can be generated at moderate laser intensities in solid-density plasmas through coherent wake emission, a process due to the coherent excitation of plasma oscillations of increasing frequencies with an increasing phase delay. Besides its fundamental interest, given the fairly high efficiency predicted $\left(\approx 10^{-4}\right)$, CWE also appears as a good candidate to obtain intense asec or fs light pulses in the $40-80 \mathrm{~nm}$ range, by using high-energy $(\approx 0.1-1 \mathrm{~J})$ laser pulses with a loose focus and typical intensities of $I \lambda^{2}=$ $10^{16}-10^{17} \mathrm{~W} \mathrm{~cm}^{-2} \mu \mathrm{m}^{2}$. The efficiency could be increased by optimizing the density gradient, to maximize the electron bunches density as well as the conversion from plasma oscillations to electromagnetic radiation.

We gratefully acknowledge the LUCA laser team for their precious help on the experiment, as well as G. Doumy, P. Salières, Y. Mairesse, and R. Marjoribanks for helpful discussions.

[1] D. von der Linde et al., Phys. Rev. A 52, R25 (1995).

[2] P. A. Norreys et al., Phys. Rev. Lett. 76, 1832 (1996).

[3] M. Zepf et al., Phys. Rev. E 58, R5253 (1998).

[4] A. Tarasevitch et al., Phys. Rev. A 62, 023816 (2000).

[5] U. Teubner et al., Phys. Rev. A 67, 013816 (2003).

[6] P. Monot et al., Opt. Lett. 29, 893 (2004).

[7] S. V. Bulanov, N.M. Naumova, and F. Pegoraro, Phys. Plasmas 1, 745 (1994).

[8] R. Lichters, J. Meyer-ter-Vehn, and A. Pukhov, Phys. Plasmas 3, 3425 (1996).

[9] Z.-M. Sheng, K. Mima, J. Zhang, and H. Sanuki, Phys. Rev. Lett. 94, 095003 (2005); Z.-M. Sheng, K. Mima, and J. Zhang, Phys. Plasmas 12, 123103 (2005).

[10] U. Teubner et al., Phys. Rev. Lett. 92, 185001 (2004); K. Eidmann et al., Phys. Rev. E 72, 036413 (2005).

[11] G. Bonnaud and G. Reisse, Nucl. Fusion 26, 633 (1986).

[12] F. Brunel, Phys. Rev. Lett. 59, 52 (1987).

[13] W.L. Kruer, The Physics of Laser Plasma Interactions (Addison-Wesley, Reading, MA, 1988).

[14] K. Varju et al., J. Mod. Opt. 52, 379 (2005).

[15] J. Mauritsson et al., Phys. Rev. A 70, 021801(R) (2004). 